\title{
Spontaneous Rupture of a Mediastinal Bronchial Artery Aneurysm Induced by Anticoagulant Agent
}

\author{
Zhimin Wang ${ }^{1}$ Chenghua Xu ${ }^{1}$ Xiaoxiao Ding ${ }^{2}$ Jinying Chen ${ }^{1}$ Huaping Xin ${ }^{1}$ \\ ${ }^{1}$ The First People's Hospital of Taizhou, Taizhou, Zhejiang, China \\ 2 The Centre Hospital of Taizhou, Taizhou, Zhejiang, China \\ Thorac Cardiovasc Surg Rep 2016;5:18-20.

\begin{abstract}
Address for correspondence Huaping Xin, MD, The First People's Hospital of Taizhou, No. 218, Hengjie Road, Taizhou No. 218, Hengji, China (e-mail: xhp691@163.com).

Jinying Chen, PD, The First People's Hospital of Taizhou, No. 218, Hengjie Road, Taizhou No. 218, Hengji, China
\end{abstract} \\ (e-mail: 498024616@qq.com).
}

\begin{abstract}
Nontraumatic spontaneous rupture of a bronchial artery aneurysm is rarely seen. In this report, we described such a phenomenon in a patient induced by usage of anticoagulant agent. The patient had no antecedent history of trauma, hypertension, or apparent aortic pathology. The patient who had been taking low-molecular-weight heparin and

Keywords

- bronchial artery aneurysm

- anticoagulant agent

- spontaneous

- rupture warfarin to treat deep vein thrombosis complained of a sudden upper abdomen pain with shortness of breath and hypoxemia. The patient was diagnosed and treated for an acute hemomediastinum caused by a ruptured bronchial artery aneurysm. If the patient had continued to take the anticoagulant antithrombotic drugs, it may cause a more virulent bleeding. Taken together, CT angiography is a useful diagnosis tool for patients with sudden chest pain and abdominal pain, and rare cause should be considered.
\end{abstract}

\section{Introduction}

Acute hemomediastinum is usually caused by rupture of a chest trauma, aorta and vertebral artery dissection, and a tumor. ${ }^{1}$ It can also occur as a result of surgery, angiography and some drug treatments. ${ }^{2,3}$ However, nontraumatic spontaneous rupture of bronchial artery aneurysm $(\mathrm{BAA})^{4}$ is very rare. There are few reports about the spontaneous rupture of a BAA caused by using anticoagulant agent of patients, who have not had any medical history of trauma, hypertension, or apparent aortic pathology. In this report, we are going to discuss a case of an acute hemomediastinum caused by a ruptured BAA. The patient in the case was using low-molecular-weight heparin and warfarin to treat deep vein thrombosis (DVT). To our knowledge, the situation in the case has not been reported in any literature yet.

received

December 7, 2015

accepted after revision

January 13, 2016

published online

April 4, 2016

\section{Case Report}

A 66-year-old man who has a medical history of pulmonary heart disease was admitted to hospital because of progressive shortness of breath and left lower extremity edema over 3 days. His initial vital signs were as follows: blood pressure (BP), 146/105 mm Hg; pulse, 94 beats/min; respiration, 20 breaths/min; and temperature, $36.5^{\circ} \mathrm{C}$. He denied he had been a tobacco user. He did not have a fever on admission. There were no signs or symptoms of radiating back pain, chest pain, orthopnea, hoarseness, or dysphagia. Laboratory test results indicated nothing abnormal (and the international normalized ratio [INR] was 1.08). A computed tomography (CT) scan of the chest showed that both sides of bronchia had signs of infection. In addition, a venous ultrasound showed there was DVT on his left lower extremity. Therefore, the patient was treated for venous thrombosis in hospital with

\footnotetext{
(c) 2016 Georg Thieme Verlag KG Stuttgart · New York
}

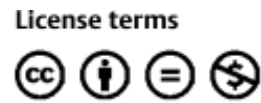


low-molecular-weight heparin $(4,100 \mathrm{U} / \mathrm{d}$ by hypodermic injection for 3 days) and warfarin ( $3 \mathrm{mg} / \mathrm{d}$ orally for a longer period of time).

With this treatment, the patient showed some improvements of his symptoms: no more shortness of breath and reduced lower extremity edema. On the 10th day of this treatment, the patient complained of sudden upper abdomen pain. He also started to vomit and cold sweating. After he was assessed, he was afebrile. BP was $83 / 44 \mathrm{~mm} \mathrm{Hg}$, and heart rate was 89 beats/min. Electrocardiogram indicated completed right bundle branch block. Arterial blood gas analysis showed that on $100 \%$ oxygen, potentiel d'hydrogene $(\mathrm{pH})$ was 7.34 , partial pressure of oxygen $\left(\mathrm{PO}_{2}\right)$ was $77 \mathrm{~mm} \mathrm{Hg}$, and partial pressure of carbon dioxide $\left(\mathrm{PaCO}_{2}\right)$ was $55.1 \mathrm{~mm} \mathrm{Hg}$. The patient had obvious stridor, and his chest was bilateral wheezing. Laboratory test results indicated nothing abnormal, except for an elevated D-dimer level of 2.05 $\mu \mathrm{g} / \mathrm{mL}$ (reference range, $0-0.5 \mu \mathrm{g} / \mathrm{mL}$ ) and INR of 2.05. CT scan of the chest showed mediastinal bleeding ( - Fig. 1 B). CT of the pulmonary artery revealed mediastinal hematoma (-Fig. 1 D-F). Aortic artery CT demonstrated a rupture on the right bronchus artery. At last, a selective arteriography (- Fig. 1G) further confirmed that there was an acute bilateral hemothorax secondary to a ruptured BAA of the right bronchial artery. The bleeding was stopped after discontinuation of lowmolecular-weight heparin and warfarin. Therefore, a rupture of the right bronchial artery with mediastinal hematoma formation was diagnosed. Then the bronchus artery aneurysm was successfully treated by coil embolization (- Fig. 1H). Finally, the patient was turning better.

\section{Discussion}

In most cases of nontraumatic mediastinal hemorrhage, there are signs of chest pain or dyspnea. However, hemorrhagic shock is rare. ${ }^{1}$ Moreover, a ruptured BAA is a rare etiology for mediastinal hemorrhage. ${ }^{1}$ BAA is a rare entity that is observed in less than $1 \%$ of all cases of selective bronchial arteriography. ${ }^{5}$ Interestingly, our patient had a sudden upper abdomen pain, hypoxemia, and shock after taking an anticoagulant agent. In this case, the initial misdiagnosis was mainly due to the clinical presentations of a sudden upper abdomen pain, worsened shortness of breath, hypoxemia, and DVT of the left lower extremity, which are also the most common symptoms and risk factors among patients with initial pulmonary embolism.

We systematically reviewed the literature for "hemomediastinum" and "ruptured BAA" on PubMed. Since the first case reported by Pugnale in 2001, ${ }^{6}$ only nine additional cases have been reported until 2014. ${ }^{7}$ In the pathogenesis of BAAs, chronic inflammation of the lung is an important contributing factor (tuberculosis, histoplasmosis, aspergillosis, bronchiectasis, etc.). ${ }^{8,9}$ In our case, the cause was likely the chronic inflammation of bronchiectasis. Unfortunately, anticoagulant agent can induce spontaneous rupture of the right BAA, and then results in hemomediastinum.

BAAs are typically classified anatomically, either as mediastinal or intrapulmonary, according to their different associated clinical symptoms. Intrapulmonary aneurysms often present with massive or intermittent hemoptysis. Although mediastinal

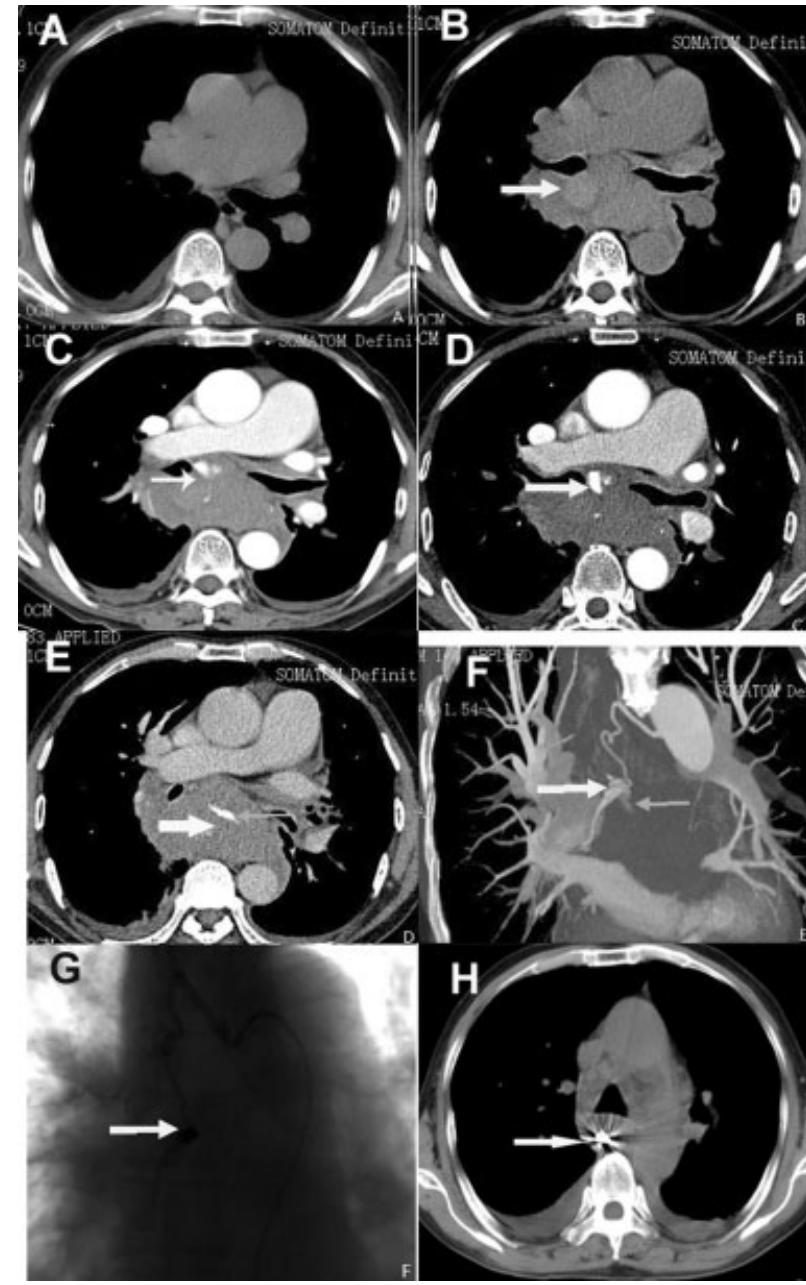

Fig. 1 (A) There was no mediastinal bleeding in the CT scan of the chest when the patient was admitted. (B) There was a mediastinal bleeding in the CT scan after the patient experienced sudden upper abdomen pain. (D-F) Aortic artery CT demonstrated a rupture on the right bronchus artery. (G) A selective arteriography further confirmed that there was an acute bilateral hemothorax secondary to a ruptured bronchial artery aneurysm of the right bronchial artery. $(\mathbf{H})$ The bronchus artery aneurysm was successfully treated by coil embolization.

aneurysms may manifest as a mediastinal mass, acute superior vena cava obstruction, dysphagia, hemothorax, hemomediastinum, and hematemesis can occur. ${ }^{10}$ On imaging, hemothorax and hemomediastinum are the most common findings ${ }^{1}$; CT angiography is the primary noninvasive diagnostic modality for BAAs. ${ }^{11}$ However, conventional angiography can be performed for both diagnostic and treatment purposes. ${ }^{5}$ Nowadays, endovascular techniques such as transcatheter embolization has been increasingly applied.

In summary, here we reported a rare case of ruptured BAA, which has not been described before. Correct diagnosis of this problem can be obtained by CT angiography and selective arteriography. This patient had a history of DVT. Therefore, when he suddenly had abdominal pain, we spontaneously assumed that he had pulmonary embolism, while ignoring other causes. If the patient had continually taken anticoagulant antithrombotic drugs, a virulent bleeding would have 
20 Anticoagulant Agent-Induced Rupture of a Mediastinal BAA Wang et al.

occurred. Taken together, common presentation is chest pain, which may lead to confusion with other syndromes, such as chest wall diseases and digestive system diseases, so CT angiography is necessary for differential diagnosis of chest pain and abdominal pain.

\section{Conflict of Interest}

The authors declare that there is no conflict of interest.

\section{Funding}

This work was not supported by any funding.

\section{Reference}

1 Seo YH, Kwak JY. Spontaneous hemomediastinum and hemothorax caused by a ruptured bronchial artery aneurysm. Korean J Thorac Cardiovasc Surg 2011;44(4):314-317

2 Taillé C, Fartoukh M, Houël R, Kobeiter H, Rémy P, Lemaire F. Spontaneous hemomediastinum complicating steroid-induced mediastinal lipomatosis. Chest 2001;120(1):311-313

3 Yokoyama H, Ohmi M, Sadahiro M, Shoji Y, Tabayashi K, Moizumi Y. Spontaneous rupture of the thoracic aorta. Ann Thorac Surg 2000; 70(2):683-689
4 Matsuge S, Hosokawa Y, Murakami Y, Satoh K. [Thymoma with idiopathic thrombocytopenic purpura preceded by mediastinal hemorrhage; report of a case]. Kyobu Geka 2002;55(10): 899-902

5 Mizuguchi S, Inoue K, Kida A, et al. Ruptured bronchial artery aneurysm associated with bronchiectasis: a case report. Ann Thorac Cardiovasc Surg 2009;15(2):115-118

6 Pugnale M, Portier F, Lamarre A, et al. Hemomediastinum caused by rupture of a bronchial artery aneurysm: successful treatment by embolization with N-butyl-2-cyanoacrylate. J Vasc Interv Radiol 2001;12(11):1351-1352

7 Vosse BA, van Belle AF, de Vries GJ, Das M. Hemomediastinum due to spontaneous rupture of a mediastinal bronchial artery aneurysm-a rare cause of thoracic pain. Respir Med Case Rep 2014; $12: 27-29$

8 Shih SY, Hu SY, Tsan YT, Lin TC. Spontaneous hemomediastinum and hemothorax caused by ruptured bronchial artery aneurysm. Eur J Cardiothorac Surg 2009;35(6):1076

9 Chatterjee A, Ghosh S, Salhiyyah K, Gaines P, Rocco G. A rare presentation of a ruptured bronchial artery aneurysm. Thorax 2004;59(10):912

10 Wilson SR, Winger DI, Katz DS. CT visualization of mediastinal bronchial artery aneurysm. AJR Am J Roentgenol 2006;187(5): W544-5

11 Hoffmann V, Ysebaert D, De Schepper A, Colpaert C, Jorens P. Acute superior vena cava obstruction after rupture of a bronchial artery aneurysm. Chest 1996;110(5):1356-1358 